\title{
PREVALÊNCIA E PERFIL SOCIODEMOGRÁFICO DE DIABETES E HIPERTENSÃO EM INDIVÍDUOS DO SISTEMA DE INFORMAÇÃO DA ATENÇÃO BÁSICA
}

\author{
Prevalence and sociodemographic profile of diabetes and \\ hypertension in individuals registered in the Primary \\ Healthcare Information System
}
Prevalencia y perfil sociodemográfico de la diabetes e hipertensión de individuos del sistema de información de la atención básica

Artigo Original

\section{RESUMO}

Objetivos: Verificar a prevalência e o perfil sociodemográfico de indivíduos com diabetes mellitus (DM) e hipertensão arterial sistêmica (HAS), cadastrados no Sistema de Informação da Atenção Básica (SIAB) do município de Lajeado-RS, no ano de 2012. Métodos: O presente estudo, transversal, descritivo-exploratório, com abordagem quantitativa, utilizou dados secundários extraídos do SIAB de 757 indivíduos. Os dados foram processados e analisados com software SPPS versão 20.0. Resultados: Da amostra de 757 indivíduos, obteve-se uma média de idade de 40,87 $\pm 16,15$ anos, sendo 50,2\% (380) do sexo masculino e 49,8\% (377) do sexo feminino. Da mesma forma, 97\% (734) são alfabetizados. Encontrouse uma frequência de apenas 3,1\% (1.131) de DM na população total e de 4,5\% (34) na amostra selecionada, e obteve-se uma prevalência de HAS de 15\% (5.849) e 17,3\% (131), respectivamente. Houve correlação positiva - fraca, mas significativa - entre HAS, DM e idade $(\mathrm{p}=0,000)$. Conclusão: Os dados obtidos indicaram uma baixa prevalência de DM e HAS em indivíduos cadastrados no SIAB em um município do interior do Rio Grande do Sul.

Descritores: Hipertensão; Diabetes Mellitus; Prevalência.

\section{ABSTRACT}

1) Univates - Lajeado (RS) - Brasil

Objective: To determine the prevalence and sociodemographic profile of individuals with diabetes mellitus (DM) and systemic arterial hypertension (SAH), registered in the Primary Healthcare Information System (Sistema de Informação da Atenção Básica - SIAB) in the city of Lajeado, Rio Grande do Sul, in 2012. Methods: This cross-sectional, descriptive exploratory study, with a quantitative approach, used secondary data retrieved from the SIAB, regarding 757 individuals. Data was processed and analyzed with SPSS software version 20.0. Results: In the sample of 757 subjects, a mean age of $40.87 \pm 16.15$ years was obtained, with 50.2\% (380) male and 49.8\% (377) female. Similarly, 97\% (734) are literate. The study found a DM frequency of only $3.1 \%(1,131)$ in the total population, and of $4.5 \%$ (34) in the selected sample, and a prevalence of hypertension of $15 \%(5,849)$ and $17.3 \%$ (131), respectively. There was a positive, weak but significant, correlation between SAH, DM and age $(p=0.000)$. Conclusion: The data obtained indicates a low prevalence of DM and SAH in individuals registered in SIAB in a countryside city of Rio Grande do Sul.

\author{
Adriane Pozzobon ${ }^{(1)}$ \\ Jairo Luís Hoerlle ${ }^{(1)}$ \\ Ioná Carreno ${ }^{(1)}$
}

Descriptors: Hypertension; Diabetes Mellitus; Prevalence.

Recebido em: $12 / 02 / 2014$

Revisado em: 03/05/2014

Aceito em: 18/07/2014 


\section{RESUMEN}

Objetivo: Verificar la prevalencia y el perfil sociodemográfico de individuos con diabetes mellitus (DM) y hipertensión arterial sistémica (HAS) registrados en el Sistema de Información de la Atención Básica (SIAB) del municipio de Lajeado-RS, en el año de 2012. Métodos: El presente estudio transversal, descriptivoexploratorio con abordaje cuantitativo utilizó datos secundarios de 757 individuos extraidos del SIAB. Los datos fueron procesados $y$ analizados en el software SPPS versión 20.0. Resultados: De la muestra de 757 individuos, se obtuvo una media de edad de 40,87 $\pm 16,15$ años, siendo el 50,2\% (380) del sexo masculino y el 49,8\% (377) del sexo femenino. De la misma manera, el 97\% (734) son alfabetizados. Se encontró una frecuencia de solo el 3,1\% (1.131) de DM en la población total y del 4,5\% (34) en la muestra seleccionada y se obtuvo una prevalencia de HAS del 15\% (5.849) y el 17,3\% (131), respectivamente. Hubo correlación positiva - débil, pero significativa - entre HAS, DM y edad ( $p=0,000)$. Conclusión: Los datos obtenidos indicaron baja prevalencia de DM y HAS en individuos registrados en el SIAB de un municipio del interior de Rio Grande do Sul.

Descriptores: Hipertensión; Diabetes Mellitus; Prevalencia.

\section{INTRODUÇÃO}

A idade cronológica é um fator preditivo para o aparecimento de doenças crônicas não transmissíveis (DCNT), tais como diabetes mellitus (DM) e hipertensão arterial sistêmica (HAS). O estudo sobre as DCNT se faz pertinente na medida em que essas patologias estão presentes no cotidiano dos serviços de saúde no Brasil e exigem deles um acompanhamento contínuo e de longo prazo da população nessa condição ${ }^{(1)}$.

A HAS é uma doença frequente na população brasileira $^{(2,3)}$, contudo, sua prevalência no país permanece desconhecida ${ }^{(4)}$. Estudos epidemiológicos apontam que níveis elevados de pressão arterial aumentam o risco de doença vascular encefálica, doenças arteriais coronarianas, insuficiência cardíaca congestiva e acidente vascular ${ }^{(5-9)}$. Além disso, a influência da HAS sobre o desenvolvimento das doenças cardiovasculares (DCV) exige o reconhecimento de sua real distribuição nos distintos estados brasileiros ${ }^{(10)}$.

De acordo com a V Diretriz Brasileira de Hipertensão Arterial, de 2006, utilizando-se o critério atual para o diagnóstico de hipertensão arterial - pressão arterial sistólica (PAS) $\geq 140$ e/ou Pressão arterial diastólica (PAD) $\geq 90 \mathrm{~mm} \mathrm{Hg}$-, as taxas de prevalência na população urbana adulta brasileira em estudos selecionados variam de $22,3 \%$ a $44 \%$, estimando-se valores entre $15,0 \%$ e $47,8 \%$ para os homens, e $15,0 \%$ e $41,1 \%$ para as mulheres ${ }^{(11)}$.
Um estudo realizado nas cinco regiões geográficas brasileiras, entre 2006 e 2010, verificou prevalência de HAS de mais de $50 \%{ }^{(12)}$. Em 2001, uma pesquisa fez o primeiro levantamento de casos suspeitos de diabetes e hipertensão pelos serviços públicos de saúde no Brasil. Participaram 4.446 municípios, que enviaram dados ao Ministério da Saúde, sendo testados 20 milhões de pessoas, detectando entre elas 3,3 milhões de casos suspeitos de diabetes ${ }^{(13)}$. Outro estudo de base populacional mostrou a prevalência conforme o sexo em diversos municípios do Brasil (no estado do Rio Grande do Sul, a prevalência ficou em 31,6\% para ambos os sexos) ${ }^{(14)}$.

$\mathrm{O}$ diabetes mellitus se configura hoje como uma epidemia mundial, sendo um grande desafio para os sistemas de saúde de todo o mundo. Estima-se que, em 2030, 11,3 milhões de pessoas serão diagnosticadas com diabetes no Brasil ${ }^{(15)}$. Deve-se levar em consideração que o diabetes conduz a muitas complicações. Um estudo que avaliou 357 pacientes diabéticos no estado de São Paulo demonstrou que metade dos pacientes não apresentava retinopatia diabética; além disso, $53,2 \%$ não sabiam qual o seu tipo de diabetes e somente $3,6 \%$ participavam de algum programa de educação sobre diabetes ${ }^{(16)}$.

Estima-se uma prevalência de DM de 7,6\% na população adulta ${ }^{(17)}$, enquanto a HAS acomete $35 \%$ da população de 40 anos ou mais no $\mathrm{Brasil}^{(18)}$. Um estudo realizado em uma Unidade de Saúde da Família de um município no interior da Bahia, em 2005, verificou que $40 \%$ da população avaliada portava pelo menos um tipo de doença crônica degenerativa, dos quais $29 \%$ possuíam HAS e $4 \%, \mathrm{DM}^{(19)}$. Indivíduos com DM e HAS precisam de mais recursos para o cuidado com a saúde, além de outros custos associados à dor, ansiedade e menor qualidade de vida para os doentes e suas famílias.

O conhecimento atualizado das condições de saúde da população advém da realização de estudos e análises das informações disponíveis, principalmente do conjunto de indicadores básicos selecionados para acompanhamento. A Política Nacional da Atenção Básica (PNAB) aponta atribuições comuns às Equipes de Saúde da Família (ESF), como: participação no processo de territorialização; integralidade da atenção a partir da promoção à saúde; atividades de planejamento e avaliação das ações da equipe através da utilização de dados disponíveis e da garantia da qualidade de registros nos sistemas nacionais de informação utilizados na Atenção Básica, dentre outras ${ }^{(20)}$. Com a expansão do número de ESF e, consequentemente, do número de famílias acompanhadas, o Sistema de Informação da Atenção Básica (SIAB) tem sido útil para condensar os dados que anteriormente eram coletados e arquivados manualmente, e por tal razão mostravam-se insuficientes, dificultando sua tradução em informações ${ }^{(21)}$. 
O SIAB foi criado no ano de 1998, pelo Departamento de Informação e Informática do SUS (DATASUS), para auxiliar o acompanhamento e a avaliação das atividades realizadas pelos agentes comunitários de saúde (ACS). Ele fornece indicadores populacionais (morbidade, mortalidade e de serviços) de uma determinada área de abrangência. Propõe, com isso, que se conheçam as condições de saúde dessa população adstrita, bem como os fatores determinantes do processo saúde-doença. Permite obter informações sobre cadastros de famílias, condições de moradia e saneamento, situação de saúde, produção e composição das equipes de saúde ${ }^{(22)}$.

A carga econômica dessas doenças produz elevados custos para os sistemas de saúde e da previdência social, devido à mortalidade e invalidez precoces, e, sobretudo, para a sociedade, as famílias e as pessoas portadoras de doenças crônicas. O Ministério da Saúde vem adotando estratégias e ações para reduzir o ônus das doenças cardiovasculares e da diabetes mellitus na população brasileira. Além disso, devese destacar a promoção da saúde, ou seja, as ações sobre os determinantes sociais que visam à melhoria da qualidade de vida. Para tanto, estudos que analisem o perfil da população assistida por serviços de assistência podem contribuir com ações intricadas no conceito de promoção da saúde, como a ampliação da consciência sanitária, a educação para a saúde, estilos de vida etc.

Nesse contexto, o presente estudo teve como objetivo verificar a prevalência e o perfil sociodemográfico de indivíduos com DM e HAS, cadastrados no SIAB do município de Lajeado-RS, no ano de 2012.

\section{MÉTODOS}

Estudo transversal, descritivo-exploratório, com abordagem quantitativa, para o qual se utilizaram dados secundários extraídos do SIAB do município de LajeadoRS, que tem aproximadamente 70 mil habitantes ${ }^{(23)}$, sendo 95\% de área urbana. O município tem nove Estratégias de Saúde da Família e conta com Agentes Comunitários de Saúde, o que resulta em uma cobertura pelo SIAB de $65,5 \%$ da população cadastrada em $2012^{(22)}$.

As equipes de ESF contam com vários profissionais que realizam treinamentos $\mathrm{e} o$ acompanhamento do preenchimento das fichas do SIAB pelos ACS. O município também tem um serviço de informática que oferece suporte específico ao registro das fichas e monitoramento do SIAB. $\mathrm{O}$ preenchimento das fichas de levantamento de dados do SIAB começa com o trabalho dos ACS. Inicialmente, os agentes cadastram todas as residências de sua área de abrangência, incluindo casas em construção e casas vazias. A ficha A representa a ficha de cadastro familiar e contém dados básicos de características socioeconômicas, de saúde e moradia das famílias e seus indivíduos, ou seja, é possível fazer um levantamento das características da população da área de abrangência do agente. Feito o cadastro inicial, os ACS devem acompanhar as famílias mensalmente, com as informações adicionais inseridas em outra ficha, a ficha B, utilizada para o acompanhamento domiciliar de grupos prioritários, como hipertensos, diabéticos, gestantes, hansenianos e tuberculosos. A ficha C presta-se ao acompanhamento das condições de saúde e seguimento médico de crianças menores de dois anos; e a ficha D é utilizada por toda a equipe da ESF para o registro das atividades diárias e notificação de algumas doenças ${ }^{(23)}$.

A população do estudo compreendeu os indivíduos adultos residentes e cadastrados no SIAB do município no ano de 2012, totalizando 36.662 cadastros. Para o cálculo da amostra dos indivíduos, utilizou-se o programa WinPepi 10.7; com o objetivo de estimar a prevalência de hipertensão, adotou-se a referência nacional de $23,6 \%^{(24)} \mathrm{e}$ o nível de confiança de $95 \%$, resultando em uma amostra de 699 indivíduos. Para estimar a prevalência de diabetes, o número calculado foi de 447 . Adotando a maior estimativa, mais $10 \%$ para inconsistência e $5 \%$ para prováveis perdas, atingiu-se um número mínimo de 757 indivíduos.

Após a definição do quantitativo amostral, selecionaram-se de forma aleatória, por sorteio, as fichas do SIAB. Coletaram-se as variáveis dados demográficos e sanitários, acesso aos serviços de saúde, bolsa família e diagnóstico de DM e HAS.

No presente estudo, utilizou-se o programa estatístico SPSS, versão 20.0, para processar e analisar o banco de dados do SIAB. Os resultados foram apresentados através de valores absolutos e relativos. Utilizou-se o teste de correlação de Pearson para análise das variáveis diagnósticas de DM, HAS, idade e alcoolismo. Adotou-se nível de significância de $5 \%(\mathrm{p}<0,05)$.

A Secretaria Municipal de Saúde autorizou o acesso aos dados, sendo toda a pesquisa conduzida dentro dos padrões éticos exigidos pela Declaração de Helsinque, de 1964, de acordo com a Resolução 466/2012 do Ministério da Saúde, após aprovação do Comitê de Ética em Pesquisa do Centro Universitário Univates, sob o protocolo $\mathrm{n}^{\circ}$ 1860.204.2009.

\section{RESULTADOS}

Dentre a população total avaliada no ano de $2012,49,2 \%$ $(\mathrm{n}=18.020)$ são do sexo masculino e 50,8\% $(\mathrm{n}=18.642)$, do sexo feminino, sendo a idade média de 39,44 $\pm 16,77$ anos. A maioria da população $(n=27.873)$ é alfabetizada. Dos 757 indivíduos maiores de 18 selecionados aleatoriamente, $50,2 \%(n=380)$ são do sexo masculino e 49,8\% (n=377), do sexo feminino. A idade média da amostra avaliada foi 
de $40,87 \pm 16,15$ anos, sendo $97 \%(n=734)$ alfabetizados, apresentando índices de alcoolismo, deficiência mental, gestação, hipertensão e diabetes muito próximos do total de indivíduos cadastrados (Tabela I).

Quanto à ocupação, $11,18 \%(\mathrm{n}=85)$ eram donas de casa, $10,13 \%(n=77)$ eram aposentados e $10,9 \%(n=83)$ eram dos serviços gerais, dentre outros. Com relação às condições de moradia e higiene sanitária, 83,5\% $(n=633)$ residem em casa de tijolo e 10,7\% $(\mathrm{n}=81)$, em casa de madeira. Quanto ao número de cômodos, $24,7 \%(\mathrm{n}=186)$ das residências possuem seis cômodos e $24 \%(n=182)$ possuem cinco. Ainda com relação à presença de elétrica (EELET), abastecimento de água $\left(\mathrm{ABAH}_{2} \mathrm{O}\right)$ e tratamento de água $\left(\mathrm{TTOH}_{2} \mathrm{O}\right)$, verificou-se que $99,5 \% \quad(\mathrm{n}=754)$ possuem EELET, $100 \%(\mathrm{n}=757)$ possuem $\mathrm{ABAH}_{2} \mathrm{O}-$ dos quais $92,3 \%(\mathrm{n}=700)$, na rede pública e 7,6\% $(\mathrm{n}=57)$, em poço ou nascente - e $84,7 \%(n=641)$ não possuem sistema de $\mathrm{TTOH}_{2} \mathrm{O}$.

Quanto ao destino do lixo, 99,3\% ( $\mathrm{n}=752)$ possuem coleta de lixo e apenas $0,7 \%(n=5)$ queimam ou enterram o lixo. Já com relação ao destino de fezes e urina, a maioria utiliza a fossa $(98,4 \%)(n=745)$ e uma pequena parte emprega o uso de céu aberto e sistema de esgoto, correspondendo a $0,8 \%(\mathrm{n}=6)$ cada um.

A Tabela II mostra os dados relativos ao acesso aos serviços de saúde, à presença de plano de saúde, bolsa família, hospitais ou unidades básicas de saúde (UBS) próximo da residência. Com relação ao plano de saúde, dos

Tabela I - Distribuição dos dados demográficos e diagnóstico de hipertensão arterial sistêmica (HAS) e diabetes mellitus (DM) na amostra de 757 indivíduos. Lajeado-RS, 2012.

\begin{tabular}{lcc}
\hline Variáveis & Sim & Não \\
& $\mathbf{n}(\mathbf{\%})$ & $23(3,0 \%)$ \\
\hline Alfabetização & $734(97,0 \%)$ & $750(99,1 \%)$ \\
Alcoolismo & $7(0,9 \%)$ & $744(98,3 \%)$ \\
Deficiência Física & $13(1,7 \%)$ & $748(98,85)$ \\
Gestação & $9(1,2 \%)$ & $626(82,7 \%)$ \\
Hipertensão arterial sistêmica (HAS) & $131(17,3 \%)$ & $723(95,5)$ \\
Diabetes mellitus (DM) & $34(4,5 \%)$ & \\
\hline
\end{tabular}

Tabela II - Distribuição dos dados sobre acesso aos serviços de saúde e ao Programa Bolsa Família na amostra de 757 indivíduos. Lajeado-RS, 2012.

\begin{tabular}{|c|c|c|}
\hline Variáveis & $\begin{array}{c}\text { Sim } \\
\text { n (\%) }\end{array}$ & $\begin{array}{c}\text { Não } \\
\text { n (\%) }\end{array}$ \\
\hline \multicolumn{3}{|l|}{ Acesso aos serviços de saúde } \\
\hline Plano de saúde & $294(38,3 \%)$ & $463(61,2 \%)$ \\
\hline Hospital & $255(33,7 \%)$ & $746(98,5)$ \\
\hline Unidade Básica de Saúde & $393(51,9 \%)$ & $502(66,3 \%)$ \\
\hline Farmácia & $32(4,8 \%)$ & $364(48,1 \%)$ \\
\hline Acesso ao Programa Bolsa Família & $11(1,5 \%)$ & $721(95,2 \%)$ \\
\hline
\end{tabular}

Tabela III - Relação entre a ocorrência do diagnóstico de Hipertensão Arterial Sistêmica (HAS), Diabetes Mellitus (DM) com a idade na amostra de 757 indivíduos. Lajeado-RS, 2012.

\begin{tabular}{lcccc}
\hline Variáveis & HAS & $\boldsymbol{p}$ & DM \\
$(\mathbf{r})$ & - & - & 0,373 & $\boldsymbol{p}$ \\
\hline $\mathrm{HAS}^{*}$ & 0,373 & $0,000 \#$ & - & $0,000 \#$ \\
$\mathrm{DM}^{*}$ & 0,545 & $0,000 \#$ & 0,311 & - \\
Idade & & & $0,000 \#$ \\
\hline
\end{tabular}

"HAS = Hipertensão Arterial Sistêmica; DM = Diabetes Mellitus. \#Correlação de Pearson. 
$38,9 \%(n=295)$ que possuem plano de saúde, $10,9 \%(n=83)$ possuem para um membro da família, $13,4 \%(\mathrm{n}=100)$, para 2 membros; $8,4 \%(\mathrm{n}=64)$, para 3 membros; $5 \%(\mathrm{n}=38)$, para 4 membros; e 1,1\% (n=8), para 5 membros. Com relação ao tipo de transporte utilizado, 51,9\% $(\mathrm{n}=393)$ utilizam carro e 65,4\% (495) ônibus.

Relacionando a presença de HAS à idade e à DM, observou-se uma correlação positiva fraca entre HAS e $\mathrm{DM}$, e entre idade e DM $(\mathrm{p}=0,000)$; e moderada entre idade e HAS $(p=0,000)$ (Tabela III). Entretanto, a relação entre o alcoolismo e a HAS não foi significativa $(r=0,029$, $\mathrm{p}=0,425$ ).

\section{DISCUSSÃO}

O SIAB fornece informações populacionais (morbidade, mortalidade e de serviços) de uma determinada área de abrangência, com o propósito de conhecer suas condições de saúde e os fatores determinantes do processo saúde-doença. Representa, assim, uma valiosa fonte de dados para a realização de diagnósticos, direcionando o planejamento e a avaliação de ações em saúde ${ }^{(25)}$.

O presente estudo avaliou mais de 700 indivíduos e mostrou uma distribuição uniforme entre o sexo dos usuários cadastrados, com idade média de 40 anos. A maioria da população é alfabetizada e possui um baixo percentual de alcoolismo e diabetes, contudo, o percentual de hipertensão esteve cerca de quatro a cinco vezes maior que o percentual de diabéticos. Pesquisas anteriores demonstraram uma relação inversa entre a alfabetização e a $\operatorname{HAS}^{(26,27)}$ - dados divergentes do encontrado nesta pesquisa. Além disso, outro estudo, realizado em 2011, também verificou uma prevalência de apenas $17,7 \%$ de analfabetos em uma população de indivíduos hipertensos cadastrados em uma Unidade de Saúde da Família no Piauí (28).

Desde os anos 1970, estudos locais realizados em áreas diferentes do Brasil, empregando processos amostrais e critérios diagnósticos diversos, apresentaram frequências de hipertensão em adultos, variando de $11,6 \%$ a $44,4 \%{ }^{(14)}$. Uma pesquisa mostrou uma taxa de prevalência de HAS de $12,6 \%$ em um município de pequeno porte no estado do Paraná, sendo maior no sexo feminino $(22,3 \%)$ e aumentando progressivamente conforme a faixa etária ${ }^{(29)}$.

O elevado número de pacientes com HAS neste estudo, apesar de a maior parte já estar realizando algum tipo de tratamento, pode ser devido à falta de efetividade da atenção dirigida a esses pacientes, ou pode estar ocorrendo falhas no controle de outras doenças crônicas (diabetes, dislipidemias, obesidade), podendo aumentar o risco para complicações cardiovasculares. Além disso, cabe destacar que muitos portadores de doenças crônicas também relutam em buscar auxílio nas UBS próximas logo no inicio dos primeiros sintomas, o que leva à piora do quadro e complicações secundárias.

Com relação à frequência do DM na população avaliada, os dados do presente estudo mostram baixos índices (entre 3\% e 4\%). Dados epidemiológicos do Ministério da Saúde, em um estudo denominado VIGITEL Brasil 2008, apontaram uma prevalência de 5,3\% de DM no Brasil, variando entre 2,4\% (em Palmas-TO) e 6,7\% (em Natal-RN e no Rio de Janeiro-RJ) ${ }^{(30)}$. No interior de Minas Gerais, encontrou-se uma prevalência de 5,8\% - nos homens, ficou em torno de $3,4 \%$ e nas mulheres, $8,0 \%{ }^{(31)}$. No interior do Rio Grande do Sul, verificou-se uma prevalência de $5,6 \%{ }^{(32)}$. Em contrapartida, uma pesquisa verificou que a prevalência de DM esteve acima de $10 \%$ na maioria dos estados brasileiros, com exceção apenas dos estados de Goiás, Rondônia e Roraima, no período de 2002 a 2004, e dos estados de Goiás e Rondônia, no período de 2005 a $2007^{(33)}$. Os resultados são conflitantes, visto que a Organização Mundial da Saúde (OMS) estimou para o Brasil, em 2000, 4,6 milhões de diabéticos e 113 milhões no ano de $2030^{(15)}$.

$\mathrm{Na}$ presente pesquisa, observou-se que, quanto à ocupação, a maioria é dona de casa $(11,18 \%)$, aposentada $(10,13 \%)$ ou prestadora de serviços gerais $(10,9 \%)$. Outro estudo, em um município do interior de São Paulo, também observou maior prevalência de dona de casa, aposentado ou pensionista ${ }^{(34)}$. Talvez esses dados possam estar associados com a prevalência de HAS ou DM encontrada no atual estudo, pois os indivíduos passam mais tempo em casa e realizam menos atividade física. Todavia, ao mesmo tempo, esses sujeitos também possuem mais tempo disponível para participar de grupos de prevenção e promoção de saúde, bem como para possuir um acompanhamento mais frequente.

Ainda foi encontrado um alto percentual $(38,9 \%)$ de uso de plano de saúde privativo, além do SUS, no estudo em questão. Outro estudo apresentou dado divergente: $91,8 \%$ dos entrevistados referiram utilizar o SUS, 5\% eram usuários de plano de saúde com cobertura integral e 6,4\%, com cobertura parcial. A utilização de planos privativos pode se refletir em um menor índice de morbidades e mortalidade por doenças crônicas, como DM e HAS, pois há menor redução do tempo para consulta médica ${ }^{(29)}$.

A presente pesquisa buscou correlacionar a presença de HAS com idade e DM, encontrando uma correlação fraca entre HAS e DM, e idade e DM, e moderada para idade e HAS. Em um estudo sobre fatores de risco para doenças cardiovasculares, foi verificado que a HAS associou-se ao $\mathrm{DM}$ e à dislipidemia, sendo esses os principais fatores de risco para doença cardiovascular ${ }^{(35)}$. Não foi evidenciada associação entre HAS e alcoolismo. Da mesma forma, um estudo realizado em 2007 não evidenciou associação entre 
tabagismo, ingestão de bebidas alcoólicas ou sedentarismo e a ocorrência de hipertensão arterial ${ }^{(36)}$. Contudo, a literatura já mostrou associações significantes entre HAS e idade superior a 60 anos, analfabetismo, escolaridade inferior a oito anos, sedentarismo e hábito alcoólico ${ }^{(37)}$.

Considerando que o SIAB é o atual meio de vigilância e acompanhamento de famílias cadastradas, existem preocupações quanto à sua eficácia, pois poderiam estar ocorrendo algumas falhas nesse processo, como falha no preenchimento dos formulários por parte dos ACS ou equívocos nos dados das UBS. Uma das hipóteses para essas falhas seria a alta rotatividade no serviço dos agentes, resultando em um inadequado preenchimento das fichas ${ }^{(38)}$. Outras questões que podem estar relacionadas à baixa confiabilidade dos dados do SIAB referem-se a não utilização pelos demais membros da equipe e à ausência de uma prática de supervisão dos ACS. O agente pode se sentir desmotivado ao preencher fichas que a equipe não utiliza ${ }^{(39)}$.

Sendo assim, os índices de diabéticos e hipertensos encontrados podem não representar o montante da população acometida por essas patologias na região. Independentemente dos números obtidos nos diferentes estudos, nas mais diversas localidades, evidências apontam para a existência de um baixo percentual de acompanhamento para indivíduos com DM e HAS, o que pode desencadear um aumento nas complicações e comorbidades dessas enfermidades, onerando a saúde pública e conduzindo a um aumento da mortalidade no país.

O presente estudo mostra dados de prevalência de DM e HAS em um município do interior do Rio Grande do Sul, os quais estão de acordo com outros estudos realizados no país. Pesquisas que avaliem a prevalência dessas doenças, dentre outras doenças crônico-degenerativas, podem contribuir para o delineamento de estratégias e promoção da saúde.

\section{CONCLUSÃO}

Os dados obtidos indicaram uma baixa prevalência de DM e HAS em indivíduos cadastrados no SIAB em um município do interior do Rio Grande do Sul. Além disto, os resultados permitiram avaliar o perfil sociodemográfico dos usuários do Sistema Básico de Atenção à Saúde no município de Lajeado-RS, sendo a maioria de alfabetizados, aposentados, que vive em casa de alvenaria, possui abastecimento de eletricidade, água e coleta de lixo.

\section{REFERÊNCIAS}

1. Cesse E, Freese E. Características e determinantes do padrão brasileiro de ocorrência as DCNT no século XX. In: Freese E. Epidemiologia, políticas e determinantes das doenças crônicas não transmissíveis no Brasil. Recife: Ed. Universitária da UFPE; 2006. p. 47-71.

2. Lessa I, Mendonça GAS, Teixeira MTB. Doenças crônicas não transmissíveis no Brasil: dos fatores de risco ao impacto social. Bol Oficina Sanit Panam. 1996;120(5):389-413.

3. Lotufo PA. Stroke in Brazil: a neglected disease. São Paulo Med J. 2005;123(1):3-4.

4. Brandão AP, Brandão AA, Magalhães MEC, Pozzan R. Epidemiologia da hipertensão arterial. Rev Soc Cardiol Estado de São Paulo. 2003;13(1):7-19.

5. Yusuf S, Hawken S, Ounpuu S, Dans T, Avezum A, Lanas $\mathrm{F}$, et al. Effect of potentially modifiable risk factors associated with myocardial infarction in 52 countries (the INTERHEART study): case-control study. Lancet. 2004; 364(9438):937-52.

6. Mohr JP. Lacunes. Neurol Clin North Am. 1983;1:20121.

7. Hennekens $\mathrm{CH}$, Albert CM, Godfriend SL, Gaziano JM, Buring JE. Adjunctive drug therapy of acute myocardial infarction: evidence from clinical trials. N Engl J Med. 1996;335:1660-1668

8. Smith SC Jr, Blair SN, Criqui MH, Fletcher GF, Fuster V, Gersh BJ, et al. The Secondary Prevention Panel: prevention heart attack and death in patients with coronary disease. J Am Coll Cardiol. 1995;26: 292-4.

9. Wachtell K, Papademetriou V, Smith G, Gerdts E, Dahlof B, Engblom E, et al. Relation of impaired left ventricular filling to systolic midwall mechanics in hypertensive patients with normal left ventricular systolic chamber function: The Losartan Intervention for Endpoint Reduction in Hypertension (LIFE) study. Am Heart J. Durham,2004;148(3):538-44.

10. Gus I, Harzheim E, Zaslasvky C, Medina C, Gus M. Prevalência, reconhecimento e controle da hipertensão arterial sistêmica no estado do Rio Grande do Sul. Arq Bras Cardiol. 2004;83(5):424-8.

11. Sociedade Brasileira de Cardiologia, Sociedade Brasileira de Hipertensão, Sociedade Brasileira de Nefrologia. V Diretrizes Brasileiras de Hipertensão Arterial. Rev Bras Hipertens. 2006;13(4):260-312.

12. Mendes GS, Moraes CF, Gomes L. Prevalência de hipertensão arterial sistêmica em idosos no Brasil entre 2006 e 2010. Rev Bras Med Fam Comunidade [periódico na Internet]. 2014 [acesso em 2013 Jun 13].;9(32):273-8. Disponível em: http://dx.doi. org/10.5712/rbmfc9(32)795 
13. Barbosa RB, Barceló A, Machado CA. Campanha nacional de detecção de casos suspeitos de diabetes mellitus no Brasil: relatório preliminar. Rev Panam Salud Publica. 2001;10(5):324-7.

14. Passos VMA, Assis TD, Barreto SM. Hipertensão arterial no Brasil: estimativa de prevalência a partir de estudos de base poulacional. Epidemiol Serv Saúde. 2006;15(1):35-45.

15. Wild S, Roglic G, Green A, Sicree R, King H. Global prevalence of diabetes: estimates for the year 2000 and projections for 2030. Diabetes Care. 2004;27(5):104753.

16. Dias AFG, Vieira MF, Rezende MP, Oshima A, Muller MEW, Santos MEX, Serracarbassa, PD. Perfil epidemiológico e nível de conhecimento de pacientes diabéticos sobre diabetes e retinopatia diabetic. Arq Bras Oftalmol. 2010;73(5):414-8.

17. Ministério da Saúde (BR), Secretaria de Políticas de Saúde, Departamento de Ações Programáticas Estratégicas. Plano de reorganização da atenção à hipertensão arterial e ao diabetes mellitus. Brasília: Ministério da Saúde; 2002 [acesso em 2013 Jun 13]. (Série C. Projetos, Programas e Relatórios; n. 59). Disponível em: http://bvsms.saude.gov.br/bvs/ publicacoes/miolo2002.pdf

18. Ministério da Saúde (BR), Secretaria de Atenção à Saúde, Departamento de Atenção Básica. Hipertensão arterial sistêmica para o Sistema Único de Saúde [Internet]. Brasília: Ministério da Saúde; 2006 [acesso em 2013 Jun 13]. (Cadernos de Atenção Básica, n. 15). Disponível em: http://bvsms.saude.gov.br/bvs/ publicacoes/caderno_atencao_basica15.pdf

19. Coqueiro RS, Nery AA, Cruz ZV, Couto de Sá CK. Prevalência de doenças crônicas degenerativas em usuários de uma unidade de saúde da família do município de Jequié-BA. Rev Bras Promoç Saúde. 2007;20(2):92-8

20. Ministério da Saúde (BR), Secretaria de Atenção á Saúde, Departamento de Atenção Básica. Política Nacional de Atenção Básica [Internet]. $4^{\mathrm{a}}$ ed. Brasília: Ministério da Saúde; 2007 [acesso em 2013 Jun 13]. (Série E. Legislação em Saúde). Disponível em: http://189.28.128.100/dab/docs/publicacoes/pactos/ pactos_vol4.pdf

21. Ministério da Saúde (BR), Secretaria de Atenção à Saúde, Departamento de Atenção Básica. SIAB: manual do Sistema de Informação da Atenção Básica [Internet]. Brasília; 1998 [acesso em 2013 Jun 13]. Disponível em: http://189.28.128.100/dab/docs/ publicacoes/geral/manual_siab2000.pdf
22. Ministério da Saúde (BR). Departamento de Informática do SUS (DATASUS). Sistema de Informação SIAB. Brasília; 2009.

23. Governo Municipal de Lajeado. Secretaria Municipal da Saúde [Internet]. 2013 [acesso em 2013 Jun 13]. Disponível em: http://www.lajeado.rs.gov.br

24. Costa JSD, Barcellos FC, Sclowitz ML, Sclowitz IKT, Castanheira M, Olinto MTA, et al. Prevalência de hipertensão arterial em adultos e fatores associados: um estudo de base populacional urbana em Pelotas, Rio Grande do Sul, Brasil. Arq Bras Cardiol. 2007;88(1):5965 .

25. Silva AS, Laprega MR. Avaliação crítica do Sistema de Informação da Atenção Básica (SIAB) e de sua implantação na região de Ribeirão Preto, São Paulo, Brasil. Cad Saude Pública. 2005;21(6):1821-8.

26. Britto RPA, Florêncio TMTM, Costa ACS, Pinheiro ME. Baixa estatura, obesidade abdominal e fatores de risco cardiovascular em mulheres. Rev Bras Med. 2011;68(3):1-4.

27. Helena ETS, Nemes MIB, Eluf-Neto J. Avaliação da assistência a pessoas com hipertensão arterial em unidades de estratégia saúde da família. Saúde Soc [periódico na Internet]. 2010 [acesso em 2013 Jun 13];19(3):614-26. Disponível em: http://www.scielo. br/pdf/sausoc/v19n3/13.pdf

28. Nascimento ES, Branco MPFC, Moreira AKF, Hazime FA. Estratificação do risco cardiovascular global em hipertensos atendidos numa Unidade de Saúde da Família de Parnaíba, Piauí. Rev Bras Promoç Saúde. 2012;25(3):287-94.

29. Sousa LB, Souza RKT, Scochi MJ. Hipertensão arterial e saúde da família: atenção aos portadores em município de pequeno porte na região Sul do Brasil. Arq Bras Cardiol. São Paulo, 2006;87(4):496-503.

30. Ministério da Saúde (BR). Vigitel 2008: vigilância de fatores de risco e proteção para doenças crônicas por inquérito telefônico [acesso 2013 Jun 13]. Disponível em: http://portal.saúde.gov. br/portal/arquivos/pdf/ VIGITEL2008_web.pdf.

31. Fidelis LC, Moreira, OC, Teodoro BG, Oliveira CEP. Prevalência de diabetes melitus no município de Teixeiras-MG. Rev Bras Ativ Fís Saúde. 2009;14(1): 23-7.

32. Costa JSD, Olinto MTA, Assunção MCF, et al. Prevalência de Diabetes Mellitus em Pelotas, RS: um estudo de base populacional. Rev Saúde Pública. 2006;40(3):3-7. 
33. Dias JCR, Campos JADB. Diabetes mellitus: razão de prevalências nas diferentes regiões geográficas no Brasil, 2002 - 2007. Ciênc Saúde Coletiva. 2012;17(1):239-44.

34. Paiva DCP, Bersusa AAS, Escuder MML. Avaliação da assistência ao paciente com diabetes e/ou hipertensão pelo Programa Saúde da Família do Município de Francisco Morato, São Paulo, Brasil. Cad Saúde Pública. 2006;22(2):377-85.

35. Ferreira SRG, Moura EC, Malta DC, Sarno F. Freqüência de hipertensão arterial e fatores associados: Brasil, 2006. Rev Saúde Pública. 2009;43(2):98-106.

36. Jardim PCB, Gondim MRP, Monego ET, Moreira HG, Vitorino PVO, Souza WKSB, et al. Hipertensão Arterial e Alguns Fatores de Risco em uma Capital Brasileira. Arq Bras Cardiol. 2007;88(4):452-7.

37. Rosário TM, Scala LCN, França GVA, Pereira MRG, Jardim PCBV. Prevalência, Controle e Tratamento da Hipertensão Arterial Sistêmica em Nobres - MT. Arq Bras Cardiol. São Paulo, 2009;93(6):672-8.
38. Freitas FP, Pinto IC. Percepção da equipe de saúde da família sobre a utilização do sistema de informação da atenção básica - SIAB. Rev Latinoam Enferm. 2005; 13(4):547-54.

39. Silva AS, Laprega MR. Avaliação crítica do Sistema de Informação da Atenção Básica (SIAB) e de sua implantação na região de Ribeirão Preto, São Paulo, Brasil. Cad Saúde Pública. 2005;21(6):1821-8.

\section{Endereço para correspondência:}

Adriane Pozzobon

Rua Avelino Tallini, 171

Bairro: Universitário

CEP: 95900-000 - Lajeado - RS - Brasil

E-mail:pozzobon@univates.br 